\title{
Un Estudio Cualitativo Sobre el Abuso Psicológico en Parejas Intragénero: Identificación, Tipos y Explicaciones
}

\section{A Qualitative Study of Psychological Abuse in Same-Gender Couples: Identification, Types, and Explanations}

\author{
Lara Longares, Álvaro Rodríguez-Carballeira, Jordi Escartín y Sheila Garrido-Rosales \\ Universidad de Barcelona
}

\begin{abstract}
Aunque en las últimas décadas se ha incrementado la investigación sobre abuso psicológico en parejas intragénero, principalmente en países anglohablantes, en países hispanohablantes todavía es escasa. Este estudio tuvo como objetivo indagar en las experiencias de victimización y perpetración de abuso psicológico en relaciones de pareja intragénero, en las posibles explicaciones del mismo y en las dificultades experimentadas por las víctimas para identificarlo. Se realizaron 12 entrevistas semiestructuradas a personas lesbianas, gay y bisexuales residentes en España que habían experimentado abuso psicológico en parejas intragénero. A través del análisis temático de las entrevistas, se identificaron algunas estrategias de abuso psicológico empleadas: aislamiento de amistades, aislamiento en el hogar, control de la vida personal, abuso emocional, imposición del propio pensamiento y amenaza de outing a la pareja. Las personas participantes aportaron explicaciones para el abuso psicológico: influencia de la lesbofobia, bifobia y gayfobia interiorizada, concepción de la pareja como una posesión y vivencia previa de violencia. Se señalan la invisibilidad de la violencia intragénero y la visión heteronormativa de los roles en la pareja como dificultades para identificar el abuso. Este estudio amplía el conocimiento relativo a las estrategias de abuso psicológico experimentadas en parejas intragénero en el contexto español e identifica algunos de los factores influyentes sobre el fenómeno y las dificultades para identificar el abuso.
\end{abstract}

Palabras clave: LGB, victimización, perpetración, violencia en la pareja, estrategias de abuso psicológico

\begin{abstract}
Although research on psychological abuse in same-gender couples has increased in recent decades, mainly in Englishspeaking countries, it is still scarce in Spanish-speaking countries. The aim of this study was to examine experiences of victimization and perpetration of psychological abuse in same-gender relationships, possible explanations for this abuse, and the difficulties experienced by the victims in identifying it. Twelve semi-structured interviews were carried out with lesbian, gay, and bisexual people living in Spain who had experienced psychological abuse in samegender couples. Through the thematic analysis of the interviews, some psychological abuse strategies were identified: isolation from friends, isolation in the home, control over personal life, emotional abuse, imposing one's ideas, and threatening to out one's partner. Participants provided some explanations for psychological abuse: influence of internalized lesbophobia, biphobia, and gayphobia, seeing the partner as a possession, and a prior experience of violence. The invisibility of same-gender violence and the heteronormative view of gender roles in the couple are pointed out as difficulties for identifying abuse. This study expands scientific knowledge about the psychological abuse strategies experienced in same-gender couples in the Spanish context and singles out some factors that influence the phenomenon and lead to difficulties in identifying abuse.
\end{abstract}

Keywords: LGB, victimization, perpetration, intimate partner violence, psychological abuse strategies

En las últimas décadas, la comunidad científica ha mostrado mayor interés por el estudio de la violencia en relaciones intragénero (Longares, Saldaña, Escartín, Barrientos \& Rodríguez-Carballeira, 2018a). La mayor parte de los estudios sobre la temática se ha realizado con muestras anglosajonas, en su mayoría estadounidenses (Badenes-Ribera, Bonilla-Campos, Frias-Navarro, Pons-Salvador \& Monterde-i-Bort, 2016). Muchos de esos estudios se han centrado en investigar la prevalencia del fenómeno (Bartholomew, Regan, White \& Oram, 2008; Walters, Chen \& Breiding 2013), variables psicosociales influyentes en el abuso, como

Lara Longares, Álvaro Rodríguez-Carballeira y Jordi Escartín, Departamento de Psicología Social y Psicología Cuantitativa e Instituto de Neurociencias, Facultad de Psicología, Universidad de Barcelona, España; Sheila Garrido-Rosales, Departamento de Psicología Social y Psicología Cuantitativa, Facultad de Psicología, Universidad de Barcelona, España.

Este estudio ha sido parcialmente financiado por el Ministerio de Economía y Competitividad de España (PSI2016-75915-P, AEI/FEDER, UE).

La correspondencia relativa a este artículo debe ser dirigida a Lara Longares. Departamento de Psicología Social y Psicología Cuantitativa, Facultad de Psicología, Universidad de Barcelona, Passeig de la Vall d’Hebron 171, 08035 Barcelona, España.

E-mail:laralongares@gmail.com 
la LGTBfobia (lesbofobia, gayfobia, transfobia y bifobia) interiorizada (Carvalho, Lewis, Derlega, Winstead \& Viggiano, 2011) o la experimentación de violencia en pareja a lo largo de la vida (Balsam \& Szymanski, 2005), y las dificultades para su denuncia (ver Alhusen, Lucea \& Glass, 2010). Además, aunque los estudios que incluyen las experiencias de violencia en relaciones intragénero con muestras de países hispanohablantes son todavía escasos (Barrientos, Rodríguez-Carballeira, Escartín \& Longares, 2016), especialmente en el caso del abuso psicológico, existen publicaciones recientes en torno a la temática (Gómez Ojeda, Barrientos Delgado, Guzmán González, Cárdenas Castro \& Bahamondes Correa, 2017; Longares, Escartín, Barrientos \& Rodríguez-Carballeira, 2018b). Asimismo, la mayoría de los estudios previos fueron realizados usando cuestionarios estructurados, útiles para obtener datos de prevalencia del fenómeno, pero que en ocasiones pueden dificultar la comprensión del significado preciso que tiene la violencia experimentada para las personas (Longares et al., 2018b). Dada la necesidad señalada en estudios previos de estudiar la violencia en parejas intragénero mediante metodologías cualitativas (Bornstein, Fawcett, Sullivan, Senturia \& ShiuThornton, 2006; McClennen, 2005), más concretamente el abuso psicológico (Barrientos et al., 2016), y de analizar los posibles factores psicosociales influyentes sobre el fenómeno (Potoczniak, Mourot, CrosbieBurnett \& Potoczniak, 2003), este trabajo pretendió contribuir a mejorar el conocimiento sobre el tipo de estrategias de abuso psicológico experimentadas en relaciones de pareja intragénero, sobre algunos de los factores que pueden influir en la perpetración de esa violencia y sobre las dificultades para identificar tal abuso. Para ello se utilizó el análisis temático de entrevistas a personas autoidentificadas como víctimas de dicha violencia.

En Estados Unidos, los datos de la Encuesta Nacional de Violencia en la Pareja y Violencia Sexual (NISVS; Walters et al., 2013) muestran que un 63\% de mujeres lesbianas, un 76,2\% de mujeres bisexuales, un 60\% de hombres gay y un 53\% de hombres bisexuales reportaron haber experimentado abuso psicológico en una relación sexo-afectiva en algún momento de sus vidas. En España no existen datos oficiales, aunque algunos estudios han proporcionado datos de victimización. Por ejemplo, un estudio muestra que un 18,6\% de participantes lesbianas y un $11,3 \%$ de participantes gay se autoidentificaron como víctimas de abuso psicológico continuado en una pareja intragénero (Longares et al., 2018b) y un estudio con hombres gay y bisexuales españoles reportó que el $70 \%$ de ellos se consideró víctima de violencia psicológica en el último año (Ortega López, 2014).

El abuso psicológico en relaciones de pareja intragénero, de un modo semejante a las parejas de distinto género, es instrumental y busca la dominación sobre la pareja para conseguir su sometimiento (Porrúa-García et al., 2016). Estudios previos realizados principalmente con muestras norteamericanas han recogido algunas estrategias de abuso psicológico en parejas intragénero, que van desde el abuso emocional, como insultar (Matte \& Lafontaine, 2011) o amenazar a la pareja (Mason et al., 2014), a intentar aislar a la persona, dificultando su contacto con su familia o amistades o limitando a la pareja el uso del computador o del teléfono (Frankland \& Brown, 2014).

Para identificar algunas de las estrategias de abuso psicológico relatadas por las personas entrevistadas, el estudio se basó en la taxonomía elaborada por Rodríguez-Carballeira, Porrúa-García, Escartín, MartínPeña y Almendros (2014) a partir de experiencias de mujeres víctimas en parejas intergénero, que recoge estrategias de aislamiento, abuso emocional, control y manipulación de la información, control de la vida personal, imposición del propio pensamiento e imposición de un rol servil. Esta taxonomía proporciona una definición clara del abuso psicológico, así como de sus componentes. De igual modo, se identificaron aquellas estrategias específicas que suceden en parejas intragénero, como amenazar a la pareja con desvelar contra su voluntad su orientación afectivo-sexual ante otras personas (Balsam \& Szymanski, 2005; Woulfe \& Goodman, 2018).

Es también necesario mencionar que esta violencia sucede en un contexto sociocultural que estigmatiza y discrimina a las personas no heterosexuales y las expone a factores únicos de estrés, como la discriminación LGTBfóbica o la LGTBfobia interiorizada (Meyer, 2003). Asimismo, las personas lesbianas, bisexuales, gay (LBG) y heterosexuales se han socializado bajo la influencia de roles de género del sistema patriarcal (Potoczniak et al., 2003) que conciben el poder y el uso de cierta violencia como un método aceptable de resolución de conflictos (Baker, Buick, Kim, Moniz \& Nava, 2013). Esto puede influir sobre la experimentación y la perpetración de la violencia en relaciones intragénero. Asimismo, el heterosexismo y la LGTBfobia estructural pueden tener repercusiones negativas sobre las víctimas de la violencia intragénero (Alhusen et al., 2010; Potoczniak et al., 2003) y podría influir negativamente en la búsqueda de ayuda de las personas LBG víctimas de violencia intragénero (St. Pierre \& Senn, 2010). 
Finalmente, con el objetivo de contextualizar la realización del presente estudio, cabe señalar que este se llevó a cabo en España, donde en las últimas décadas se aprobaron diversas medidas jurídicas para reconocer los derechos de las personas lesbianas, gay, transexuales, bisexuales e intersexuales (LGTBI+) y los derechos de las mujeres. A este respecto, en 2005 se aprobó la Ley 13/2005 que legalizó el matrimonio igualitario, en 2004 se aprobó la Ley Orgánica 1/2004 de medidas de protección integral contra la violencia de género y también legislación autonómica contra la discriminación de las personas LGTBI+, como la Ley 11/2014 para garantizar los derechos de lesbianas, gay, bisexuales, transgéneros e intersexuales y para erradicar la homofobia, la bifobia y la transfobia del Parlamento de Cataluña.

Por todo ello, el presente estudio se propuso identificar las estrategias de abuso psicológico experimentadas en parejas intragénero, los posibles factores identificados como explicativos de la perpetración del abuso y las dificultades encontradas para identificar el abuso.

\section{Método}

\section{Diseño}

El presente estudio se realizó a partir del análisis cualitativo de entrevistas semiestructuradas.

\section{Participantes}

Para la participación en el estudio se incluyeron personas mayores de 18 años que hubieran tenido una o más relaciones sexo-afectivas conflictivas con personas del mismo género. Un total de 12 personas de entre 22 y 34 años de edad participaron en el estudio.

Se difundió el estudio como una investigación cuyo objetivo era explorar la violencia vivida a lo largo de la vida por personas LGB y, más concretamente, aquella vivida en el contexto de una relación de pareja. Las personas fueron contactadas mediante la estrategia de bola de nieve, a través de contactos personales y de las redes sociales.

De las personas participantes (en adelante se hará alusión a "las participantes" para referirse a las personas que han participado en el estudio), cinco se identificaron como mujeres lesbianas, uno como hombre gay, uno como hombre homoflexible y cinco como bisexuales (3 mujeres y 2 hombres). Todas las participantes en el estudio se identificaron como víctimas de violencia psicológica. Todas residían en España: la gran mayoría residía en núcleos urbanos de la provincia de Barcelona (10 participantes), una persona residía en la comunidad autónoma de Madrid y otra en Galicia. Una amplia mayoría tenía estudios universitarios o técnicos (8), dos participantes tenían estudios secundarios o medios, y dos participantes tenían estudios de postgrado. En relación a la situación profesional, la mitad de las participantes eran estudiantes que en su mayoría combinaban estudios con trabajo remunerado. La mayoría de las participantes trabajaba en la empresa privada, la administración pública o como autónomas y dos no tenían empleo.

\section{Instrumento}

Se realizaron entrevistas semiestructuradas de forma individual con cada participante del estudio. Las entrevistas fueron realizadas por una investigadora formada en investigación cualitativa y realización de entrevistas. Las entrevistas incluyeron experiencias de violencia vividas a lo largo de la vida y las dificultades para identificar el abuso en parejas intragénero y denunciarlo. En el presente estudio, se han incluido dos bloques temáticos: violencia experimentada en relaciones sexo-afectivas con personas del mismo género y dificultad de identificar el abuso en parejas intragénero y denunciarlo. En el bloque temático de violencia experimentada en relaciones intragénero, se preguntó a las participantes por sus experiencias de abuso, para conseguir un relato detallado que incluyera las estrategias experimentadas y perpetradas de abuso, la intencionalidad de dichas estrategias y quién las llevaba a cabo. En el segundo bloque temático se incluyeron preguntas para identificar las fuentes de apoyo principales y las dificultades encontradas a nivel de apoyo formal e informal que permitieron conocer las dificultades para identificar el abuso en parejas intragénero y denunciarlo. 


\section{Procedimiento}

Las entrevistas se llevaron a cabo entre febrero y julio de 2017 y se realizaron de forma presencial con las personas que residían en la provincia de Barcelona y mediante videoconferencia (vía Skype) con las dos personas que residían en Madrid y Galicia.

Las entrevistas se concertaron según la disponibilidad de tiempo de las participantes. Estas personas fueron informadas de que su participación en el estudio sería anónima y confidencial, de acuerdo a la normativa legal vigente, y firmaron un documento de consentimiento informado. Las participantes no recibieron ninguna compensación económica ni de ningún otro tipo por su participación en el estudio. Con el objetivo de anonimizar el estudio y procurar la confidencialidad de los datos, los nombres de las participantes fueron modificados en la transcripción de las entrevistas, así como los nombres de personas que aparecían mencionadas durante las entrevistas.

Todas las entrevistas realizadas fueron registradas en formato audio y posteriormente transcritas para su análisis. Algunas de las entrevistas fueron realizadas en castellano y otras en catalán, según la preferencia de las participantes. Para este artículo, las entrevistas realizadas en catalán han sido traducidas por una investigadora bilingüe.

\section{Análisis de Datos}

Se llevó a cabo un análisis temático con el contenido transcrito de las entrevistas, siguiendo las recomendaciones de Braun y Clarke (2006) y Vaismoradi, Turunen y Bondas (2013), mediante el software Atlas-ti versión 7.5. En la primera fase del análisis, se revisó el contenido de todas las entrevistas de forma intensiva para identificar los temas principales, con el objetivo de crear un sistema de códigos para estructurar el análisis. Este sistema combinó códigos apoyados en investigaciones previas con códigos de nueva creación, a partir de los temas surgidos en las lecturas de las entrevistas, empleando una combinación de estrategias deductivas e inductivas para el análisis (Joffe, 2012). Más concretamente, con el objetivo de analizar las estrategias de abuso psicológico experimentadas o perpetradas por las participantes, el estudio se basó en la taxonomía elaborada por Rodríguez-Carballeira et al. (2014), que incluye definiciones operativas para cada tipo de conducta abusiva. Asimismo, los nuevos códigos se fueron creando en una combinación entre la información extraída a partir de la investigación previa y las experiencias relatadas por las participantes en el estudio.

En la segunda fase se aplicó el sistema de códigos a las transcripciones de las entrevistas y, siguiendo las recomendaciones de Braun y Clarke (2006), se revisó nuevamente el sistema de códigos, añadiendo las modificaciones necesarias. Periódicamente, el sistema de códigos se fue modificando al ir revisando la codificación de las transcripciones de las entrevistas.

\section{Resultados}

Se proporcionarán los hallazgos en tres categorías, acordes con el propósito del estudio: estrategias de abuso psicológico en parejas intragénero, explicaciones al abuso y dificultad de identificar el abuso y denunciarlo. Las tres categorías se derivan del análisis del conjunto de las entrevistas. Después de cada fragmento de entrevista, se indica entre paréntesis el género indicado por la participante (M: mujer; $\mathrm{H}$ : hombre), la edad y la orientación afectivo-sexual.

\section{Estrategias de Abuso Psicológico en Parejas Intragénero}

En el siguiente apartado, se analizan las diferentes estrategias de abuso psicológico. En primer lugar, se incluyen las estrategias contenidas en la taxonomía de Rodríguez-Carballeira et al. (2014) y, a continuación, las encontradas mediante el análisis inductivo.

Aislamiento. Separar o distanciar al otro miembro de la pareja de su círculo relacional es una estrategia que promueve el aislamiento de la persona que experimenta la violencia, contribuye a dificultar la identificación y la denuncia de la violencia, y consigue que la vida de la persona víctima del abuso gire en torno a su pareja. 
Aislamiento de las amistades y de la red social. Las participantes relatan que su pareja las aislaba de sus propias amistades y promovía que se relacionaran más con las amistades de la persona perpetradora. Un participante relata:

Pero lo fuerte es que él quedaba también con sus amigos y me decía que yo fuera con ellos. Como quería aislarme de mis amigas para que fuera con él y sus amigos, yo intentaba hablar con él, que es importante tener cada uno su espacio, pero era imposible. (H, 24, Homosexual)

Asimismo, como señala esta participante, los celos y la desconfianza hacia la pareja también aparecen ligados a la estrategia de aislamiento y como un argumento asumido como válido para impedir que la persona se relacione con otras amistades, como indica esta participante:

También es verdad que a mí no me dejaba salir con mis amigas, porque como anteriormente había sido infiel, pues lo podría volver. No me dejaba quedar ni hacer nada. Eso sí, ella salía, volvía borracha, no me contestaba las llamadas. Si ella no me deja, ella tampoco. (M, 32, Lesbiana)

Cabe destacar que la estrategia de aislamiento es descrita por algunas de las participantes como un proceso que sucede de forma gradual, como indica esta participante:

(...) y al dejar de ir de fiesta, pues ya no ves tanto a tus amigos, entonces empiezas a aislarte. Yo me aislé muchísimo cuando esta chica apareció. Luego le hablaba de mis amigos y ella me decía que uno era conflictivo, que si el otro no me gusta, que si ese te va a traer cosas malas... no me prohibió en ningún momento hacer nada, pero te las soltaba y te las soltaba y te las soltaba. Entonces, claro, vive con eso. No me di cuenta, pero entonces dejé de ir con esos amigos. Porque, claro, no le gustaban y cada vez que le hablaba de ellos era tener caras largas. (M, 24, Bisexual)

Este proceso gradual, característico de las relaciones de pareja abusivas (Porrúa-García et al., 2016), dificultaría que las personas identifiquen el comportamiento de la pareja como una estrategia para aislarlas, pudiendo, incluso, confundirse con una voluntad de proteger a la pareja de amistades que puedan resultar perjudiciales para ella.

Aislamiento del ámbito laboral, de estudios y de intereses, y en el hogar. Dos participantes relataron haber sido aisladas del ámbito laboral o a obligarlas a quedarse en el hogar. En el caso del aislamiento del ámbito laboral, de estudios y de intereses, formaba parte de la idea de aislarle de su entorno y de las actividades que realizara de un modo generalizado, con el propósito de acaparar a la persona y que su vida girara en torno a la de su pareja. Este participante lo describe así:

Daba muchísimo, pero luego, cuando hizo el cambio este que te he contado, me quería a mí completamente. Para mí y ya está, no quiero nada más. Y tampoco quiero que hagas nada. (H, 23, Homoflexible)

En el caso de la participante que fue confinada en el hogar, esta estrategia de aislamiento estaba relacionada con la ocultación de la relación, temiendo que otras personas pudieran saber que tiene una relación sexo-afectiva con otra persona de su mismo género. En este caso, el aislamiento estaría relacionado con la LBGfobia (lesbofobia, bifobia y gayfobia) interiorizada, es decir, una dificultad de aceptación de la propia orientación afectivo-sexual por parte de la persona perpetradora:

Porque luego a la tarde se iba al parque, donde yo no podía ir. Me tenía encerrada en casa. Ella me decía la excusa de que era para pasar tiempo con su hija, pero un día que fue al parque vino también su hermana. Y un día y dos... luego me enteré que el exmarido también iba al parque. Y no sé, me está prohibiendo ir a mí y luego a su exmarido no le dice nada. Un día le dije: "escucha, puede ir tu exmarido, puede ir tu hermana, ¿Qué soy yo el problema?" Y me suelta: "sí, tú. Que no quiero que tú vengas". Yo siempre se lo decía, te avergüenzas de mí. Ella me decía que era porque no quería que me vieran con ella, por lo que dirían, pensaran... Pero yo pensaba: "deja que me conozcan como Diana, no como tu pareja". Me tenía escondida como una rata. Nunca me dejó salir con ella al parque. (M, 34, Lesbiana)

\section{Control de la vida personal.}

Control sobre las actividades cotidianas y el uso del tiempo. La estrategia de mantener a la otra persona bajo vigilancia y mantener el control sobre sus actividades y uso del tiempo con la intención de que la vida de la otra persona gire alrededor de quien perpetra la violencia, ha sido experimentada por la mayor parte de las participantes (10). En la mayoría de los relatos, la pareja intenta ejercer ese control pidiendo explicaciones a la persona sobre las actividades que está haciendo, el lugar donde se encuentra y las personas con las que está, y en diversos casos ese control era ejercido mediante el teléfono móvil, bien sea a través de aplicaciones de mensajería instantánea o bien obligándole a enviarle fotografías para vigilar sus actividades, como relata este participante: 
También me hacía mandarle fotos cuando yo estaba en casa para que así él comprobara que no le estaba engañando. (H, 24, Homosexual)

Esta estrategia de control la experimentaron durante la relación de pareja y tras su ruptura. En el caso de esta participante, se vio obligada a seguir conviviendo con su expareja, que siguió perpetrando estrategias de control tras la ruptura:

Me fui a la lavandería a lavar la ropa para no molestar. Cuando vuelvo mi ex me dice que a dónde he ido y por qué había tardado tanto. Controles así, sin ser pareja ya, preguntándome dónde estaba, con quién iba, mirarme el móvil. (M, 32, Lesbiana)

Cabe destacar que algunas participantes indican que, con el objetivo de satisfacer las necesidades de la pareja y aumentar la confianza, acordaban con ella acceder a sus dispositivos móviles, hecho que suponía un mayor control sobre la vida de la pareja. Este es el relato de una participante:

Me vigilaba con quien iba o dejaba de ir, nos "dejábamos" mirar el teléfono para ver que no había ninguna conversación de alguien más... yo pensaba que eso le ayudaría, pero no. (M, 25, Bisexual)

La estrategia de control de la vida personal perpetrada durante la relación de pareja también aparece relacionada con los celos en los relatos de las participantes:

Un día quedé con una amiga para tomar algo por plaza Cataluña. Estábamos en un bar y eso que no miras tanto el móvil, lo típico que hace todo el mundo. La tía [forma de referirse a una persona] me acabó llamando, seguramente para ver dónde estaba. No le cogí el teléfono porque estaba con mi amiga y me pareció una falta de respeto. Entonces, le escribí un Whatsapp diciendo que estaba bien y que aún estaba con mi amiga. Cuando llegué a mi casa por la noche, me montó el pollo [hacer un escándalo]. La llamé y me dijo que "de qué coño iba" [expresión informal para pedir explicaciones a alguien], que sabía que le estaba engañando, que no es normal quedar tantas horas con una persona y no contestar... No me pareció normal. (M, 33, Lesbiana)

Asimismo, un participante relata que, tras recibir por parte de su pareja estrategias de control a través de los celos, en ocasiones él también perpetró esa estrategia durante la relación de pareja:

Entonces, entramos en esa espiral de celos y yo llegué a decirle cosas también, en plan "con quién estás, por qué te escondes el móvil...". Pero en parte era porque él mostraba una actitud que hacía que yo me preocupara. (H, 24, Homosexual)

Con el objetivo de ejercer ese control, algunas participantes también indicaron que su pareja les había seguido físicamente, como explica esta participante:

Hasta que volvió a ser la que era, agobiándome otra vez cuando salía con mis amigas, discutiendo otra vez por el puto trabajo, persiguiéndome, porque me seguía a ver dónde iba, también en el trabajo... ¡estaba loca, tía! (M, 33, Lesbiana)

Finalmente, una participante describe el control sobre la vida personal, tratando su pareja de cambiarle diversos aspectos para acomodarla al criterio de la perpetradora:

Era una chica que no tenía claro dónde terminaba ella y dónde empezaba yo. Entonces, cada dos por tres intentaba cambiarme. Que si esta ropa no me gusta, que si no te queda bien, que si este color de pelo no es serio, deberías volver a tu color natural... Todo, en todo tenía una opinión. (M, 24, Bisexual)

Abuso emocional. En este apartado se incluyeron aquellas acciones perpetradas para influir en los sentimientos y emociones de la otra persona, manipularla y obtener sumisión.

Menosprecio, humillación y rechazo a la otra persona. Fue la estrategia de abuso emocional descrita con mayor frecuencia por las participantes. Este menosprecio se ejercía mediante burlas, insultos o ridiculización. En la amplia mayoría de situaciones descritas el menosprecio se experimentaba durante la relación. Dos participantes también han relatado haber recibido agresiones verbales, a pesar de no haberlas concretado. Es destacable el hecho de que algunas de las estrategias de menosprecio relatadas aparecen relacionadas con los celos por parte de la pareja. En el relato de una participante, la palabra "puta", empleada como insulto hacia ella, está relacionada con los celos por parte de su pareja:

Lo que te he dicho que constantemente me llamaba puta, desagradecida, me desvaloraba también, me decía que por mucho que tuviera una carrera, era muy tonta, que no sabía nada de la vida. (M, 25, Bisexual)

Asimismo, en ocasiones este menosprecio iba dirigido a infravalorar a la persona en su trabajo o sus actividades principales, como relata este participante:

El "no vales para nada", "tu trabajo es una mierda", cosas de estas. De "no vas a hacer nada en la vida. Vas a

pasar hambre"... Estos comentarios los he tenido durante mucho tiempo. (H, 23, Homoflexible) 
El menosprecio también ha sido perpetrado por dos participantes como reacción o bien al menosprecio recibido por parte de la pareja o bien al intento de control por parte de la pareja durante la ruptura de la relación, como en el caso de esta participante:

Sí, me dio a elegir. Me dijo literalmente "el trabajo o yo". Entonces allí fue la única vez que la insulté. Recuerdo que le dije "vete a la mierda". Estaba harta, harta de todo, me iba a volver loca al final. Es muy fuerte que una persona te dé a elegir, cuando es trabajo, es dinero que necesito, que no me cae del cielo. Una persona madura y segura de sí misma no da a elegir. (M, 33, Lesbiana)

Activación interesada de emociones positivas. Esta estrategia se basaría en ofrecer expresiones calculadas de amor o hacer promesas placenteras a la otra persona después del abuso, para obtener su perdón y evitar el distanciamiento o separación. De las personas que han relatado haber experimentado esta estrategia, la mayoría de ellas la vivió durante la ruptura de la relación o en situaciones en que la pareja temía la separación, como relata esta participante:

Le dije que me había hecho daño y no sabía si podía hacer como si nada. Durante las semanas siguientes "se lo curraba" [trabajó duro], no sé, me hablaba bien, quedamos un día y no discutimos. Claro, volví a caer. Quedamos una noche y me enfatizó diciendo que había cambiado, que podríamos estar así de bien como esos días que habíamos estado... mentira, todo mentira. (M, 33, Lesbiana)

Intimidación y amenazas. La estrategia de amenazar a la otra persona se ve reflejada en los relatos de las participantes en relación a dos tipos de consecuencias negativas que la persona puede experimentar si no hace lo que dice la persona perpetradora: consecuencias físicas y consecuencias psicológicas. La amenaza con la agresión física ha sido relatada únicamente por un participante:

También me ha amenazado más con pegarme, en plan "o esto o te meto una paliza". (H, 23, Homoflexible)

Y la amenaza relatada más común, que estaba relacionada con consecuencias psicológicas negativas, era la de amenazar con dejar a la pareja para conseguir algo, como explica esta participante:

Y me amenazaba con dejarme constantemente, con ultimátum, en plan si no esto lo dejamos. Me parece una gran inmadurez. Entonces, yo como que reculaba, no entiendo por qué, pero acababa perdonándola, dándole oportunidades, porque no sé, supongo que no quería quedarme sola. (M, 33, Lesbiana)

Tres personas entrevistadas también explican que durante la relación sus parejas o ellas mismas lanzaron o rompieron algún objeto durante una discusión, y las personas entrevistadas lo identifican usualmente como una dificultad de comunicarse verbalmente en ese momento. Estos actos podrían formar parte de una estrategia de intimidación hacia la pareja, mediante actitudes amenazantes. Una de las entrevistadas relata:

Entonces, le dije una broma: que a ver si tendría una novia por ahí y no me había enterado. Era broma, no lo pensaba realmente, se lo dije porque como no me estaba haciendo caso... total que le cambió la cara. Cogió, me miró seria y me dijo "si eso es lo que crees". Se levantó, tiró la guitarra al suelo, le tiró una patada a la silla que la mandó a la pared. La chavala [muchacha] tenía fuerza porque hacía un metro ochenta, era un monstruo esa mujer. Y se fue contra la pared, no sé si se iba a dar un golpe a la cabeza... yo que sé, se le fue. (M, 24, Bisexual)

Manipulación de la culpa. Algunas participantes describen que su pareja les hacía sentir culpables por alguna actitud, comportamiento u omisión de la persona agresora. En el caso de esta participante, es culpada de tener un ataque de ansiedad por parte de su pareja, tras un episodio en que la pareja la menosprecia repetidamente:

Me calmé, pero recuerdo que ella me dijo: "todo esto te lo has buscado tu solita". (M, 25, Bisexual)

Asimismo, tres participantes relatan que la manipulación de la culpa la perpetraba la pareja mediante la amenaza de suicidarse, con el objetivo de despertar la culpabilidad en la otra persona y evitar la separación:

(...) me hacía mucho chantaje emocional cuando decía de dejarla, se ponía delante de la puerta para que no pudiera salir de casa. Me decía que se iba a matar, que se iba a suicidar, que se iba a cortar y... bueno, seis meses así. (M, 29, Lesbiana)

Despreocupación por las emociones. Dos entrevistas muestran la estrategia de mostrar indiferencia ante los sentimientos, emociones o ideas de la otra persona, tanto durante la relación como durante la ruptura. En ambos casos, la persona entrevistada se encuentra en una situación de salud mental o física delicada y sus parejas muestran esa despreocupación, tanto por sus emociones como por su salud, como relata esta participante: 
Me fui a casa, me senté delante de ella y me dijo que me dejaba. Que como no cobraba, no tenía ingresos ni nada, me dejaba. Y que ella tenía que vivir la vida y que yo estaba enferma y no era su culpa. Una de las frases que siempre me decía es "tu enfermedad no es mi responsabilidad". (M, 32, Lesbiana)

Imposición del propio pensamiento. En este tema se incluyen estrategias, en este caso únicamente perpetradas por las parejas de las personas entrevistadas, que consistían en desacreditar las ideas de la otra persona e imponer el propio punto de vista. Esta estrategia ha sido una de las menos frecuentemente relatadas en las entrevistas.

Denigración del pensamiento crítico. Uno de los participantes relata como su pareja desacreditaba y rechazaba sus razonamientos cuando no estaba de acuerdo con él:

Si decías una cosa que era contraria a lo suyo, te trataba de loco y tenía que ser lo que él dijera. Estaba demasiado obsesionado con tener razón. Esto llevaba a extremos a veces de gritarme, estar en medio de la calle y de encima pirarse [marcharse] y yo pensando "¿qué haces?". (H, 27, Bisexual)

Redefinición de la realidad. Dos participantes explicaron la estrategia en la que sus parejas rechazaban su percepción de una situación problemática, minimizando o negando cualquier responsabilidad personal y la reinterpretaban de manera interesada. Cabe destacar que en ambos casos, la redefinición de la realidad iba acompañada de manipulación de la culpa, como explica esta participante:

Luego llegó un punto que ella se volvió muy fría conmigo. Me puso los cuernos [le fue infiel], porque sé que me los puso. A veces tonteó con tías delante de mí. Yo se lo reprochaba, pero ella me decía que no estaba sucediendo, que eran imaginaciones mías. Entonces, yo me culpaba a mí misma por desconfiar de ella. Giraba las cosas para que pareciera que yo estaba loca. (M, 24, Bisexual)

Imposición de un rol servil. La imposición de la autoridad sobre la pareja, forzándola a tener un papel subordinado al servicio de las exigencias y preferencias de la persona que abusa, ha sido experimentada por dos personas entrevistadas durante la relación de pareja, como describe un participante en la entrevista:

En una semana me trató bastante mal en casa. Yo lo hacía todo y por su parte eran exigencias: "Tienes que hacer tal o lo otro", como si yo fuera la madre ¿sabes? (H, 27, Bisexual)

Las estrategias incluidas hasta el momento han sido identificadas a partir de un método deductivo. La siguiente estrategia incorporada, la amenaza de outing, y los siguientes temas introducidos en los resultados han sido determinados a partir de una estrategia inductiva, aunque teniendo en cuenta los antecedentes aportados por estudios previos.

Amenaza de outing. Esta amenaza aparece separada del resto de las amenazas en la relación de pareja, dado que se trata de una estrategia que únicamente puede experimentarse o perpetrarse en relaciones en las que por lo menos una parte de la pareja no es heterosexual. Por ello, ha sido considerada por estudios previos como una estrategia específica de violencia en relaciones intragénero (Woulfe \& Goodman, 2018). Consiste en amenazar a la pareja con desvelar su orientación afectivo-sexual ante otras personas. En este estudio, dos participantes han relatado haberla experimentado durante la relación de pareja y uno de ellos también tras la ruptura de la relación. Uno de los participantes lo explica de este modo:

Y amenazas, al principio con contárselo a mi madre, pero después ya con contárselo a mi padre, que sabía que ahí me dolería y cedería, porque mi padre no tiene que saber nada. Lo veía capaz, porque como tenía su huella en mi teléfono, entró y cogió su número y lo tenía guardado en su móvil. Entonces, cuando discutíamos, me decía que le llamaría. (H, 24, Homosexual)

De nuevo, este relato muestra como dicha estrategia puede ser perpetrada empleando las tecnologías, en este caso el dispositivo móvil que facilitaría el contacto con la familia del participante.

\section{Explicaciones del Abuso en Parejas Intragénero}

Esta categoría incluye distintos temas para los que las personas entrevistadas han aportado explicaciones o razones que atribuyen a la perpetración de la violencia, tanto por parte de su pareja como por parte de ellas mismas.

LBGfobia interiorizada. Casi la mitad de las participantes identificaron las dificultades que tenían sus parejas en la aceptación de su propia orientación afectivo-sexual como una explicación clave a la perpetración de la violencia por parte de la/s pareja/s. Una de las participantes explicaba: 
Yo creo que odiaban sentir algo por mí, que me querían, pero odiaban hacerlo, odiaban estar con una mujer. Por ejemplo, este último caso de la chica, pues creo que era su mayor deseo, pero a la vez se despreciaba a sí misma por sentir algo por una mujer. No se aceptaba. A lo mejor la sociedad espera que estés con un hombre, crees una familia... entonces eso le rayaría [le preocuparía]. (M, 27, Bisexual)

Estos problemas de aceptación, según estos relatos, promoverían que rechazaran también a sus parejas. De hecho, tres participantes relataron que su pareja no aceptaba su relación, por ser una relación intragénero, y lo identificaron como un acto de hostilidad por parte de su pareja, como indica esta participante:

(...) todo fue psicológico, sobre todo peleas constantes y el hecho de que se avergonzara y no aceptara nuestra relación. (M, 28, Lesbiana)

Asimismo, la entrevistada identificaba en su pareja un rechazo hacia su propia orientación afectivosexual (que no se especifica durante la entrevista) que, a su vez, entendía como una discriminación hacia sí misma:

Ella misma se discriminaba y, por lo tanto, también me estaba discriminando a mí. Si ella no se acepta, quiere decir que a mí tampoco me acepta. (M, 28, Lesbiana)

Concepción de la pareja como posesión. La idea de la pareja como una posesión y los celos que siente la pareja forman parte de una de las ideas explicativas en las que han coincidido casi la mitad de las personas entrevistadas. Uno de los participantes expresa así la idea de las actitudes posesivas por parte de su pareja:

Por su actitud posesiva, quería que estuviera con él siempre. Al principio muy bien, porque de alguna forma quieres mostrarte bien a la persona, pero poco a poco, aunque fue muy rápido, esas ideas posesivas y actitud controladora hicieron que actuara así. (H, 24, Homosexual)

En diversos casos, relacionan esta actitud posesiva con las estrategias de control hacia la pareja. Asimismo, los celos han sido también una frecuente explicación de la perpetración de estrategias de abuso psicológico, como expone esta participante:

Los celos. Me considero una persona que le gusta hablar las cosas. Intento que nunca se cree un mal clima, pero se acaba creando porque pasa de forma natural. Intento relajar la situación y tal, más el tema de los celos ha podido ser uno de los desencadenantes de conflictos que he tenido con mi pareja. Se ponía celoso incluso de mis amigas. (H, 24, Homosexual)

Vivencia previa de violencia. La vivencia en un ambiente familiar con violencia y el hecho de haber sufrido bullying han sido identificadas por tres participantes como explicaciones de la perpetración de violencia por parte de sus parejas. De un lado, una de las explicaciones atribuidas al vínculo entre la violencia vivida a lo largo de la vida y la posterior violencia perpetrada era la normalización del ejercicio de la violencia, como relata esta participante:

(...) yo creo que su situación era muy conflictiva y además ella había vivido mucha violencia en su casa. Ella tenía una madre maltratadora, esto es un hecho. Si a ti te maltratan, ese maltrato se normaliza. (M, 24, Bisexual)

Asimismo, y en relación al aprendizaje de la violencia como forma de relacionarse, algunas participantes relatan haber ejercido el abuso como forma de defensa ante otra posible experiencia de violencia, como señala este participante:

Yo creo que él, al haber sufrido tanto bullying, tanto abuso, era una persona que a la mínima se ponía a la defensiva o se enfadaba. (H, 27, Bisexual)

Algunas participantes identificaron también el actuar a la defensiva por temor a ser dañada como posible explicación a la perpetración que, en ocasiones, como en el relato de este participante, estaba relacionado también con experiencias previas de malos tratos:

Tenía miedo a que yo no me lo tomara en serio o algo así. Sé que en sus relaciones anteriores lo pasó mal, porque todos como que pasaban de él e incluso lo llegaron a tratar mal. Y no sé si eso hizo que de algún modo me tratara así a mí. (H, 24, Homosexual)

Relativas a la salud mental de la persona perpetradora. Las referencias a la salud mental de la persona que perpetra la violencia, a su autoestima, sus inseguridades y sus miedos son mayoritarias entre las explicaciones aportadas por las personas entrevistadas. En algunos casos, estas hacían referencia a una baja autoestima por parte de la pareja, lo que le dificultaba aceptarse a sí misma, como relata esta participante:

Eso era porque era una insegura, tenía problemas de autoestima, aceptarse a sí misma y de algún modo eso lo pagó conmigo, pero, bueno, que estuviera mal no justifica el infierno que viví por ella. (M, 25, Bisexual) 
Asimismo, cabe destacar que la participante no identifica la posibilidad de encontrar una explicación para la violencia con la justificación moral de dicha violencia.

Tres participantes destacan también como explicación a la perpetración del abuso psicológico la voluntad de hacer daño a la pareja. De hecho, una de las entrevistadas atribuye a la perpetradora un sufrimiento y una voluntad de extender ese sufrimiento hacia la pareja:

Era una persona muy tóxica y también quería hacerme sentir mal a mí, para que sufriera como ella. (M, 33, Lesbiana)

Interés socioeconómico. Dos participantes señalaron que una de las explicaciones a la violencia era por intereses socioeconómicos. Una de las participantes señala:

Para quedarse con el piso [vivienda]. Claro es que... hay cosas que no se entienden, porque me dice que no quería hacerme daño... pero después decirme que le doy asco y cosas que me hacen daño... pues, no se entiende. Entonces, sé que parte es por el piso. (M, 32, Lesbiana)

Es destacable el relato de otra participante que muestra la influencia de las condiciones socioeconómicas sobre la perpetración del abuso, y cómo estas se ven atravesadas por el estigma asociado a la orientación afectivo-sexual que hace que exponer públicamente dicha orientación pueda implicar riesgos:

Yo creo que si yo hubiera tenido dinero, hubiera sido diferente. Yo creo que era del palo [expresión similar a "como"] "estoy con ella y si tiene dinero no me importa que me vean". Pero, yo como no tengo dinero, ¿por qué voy a dar el paso a que me vean? (M, 34, Lesbiana)

\section{Dificultad de Identificar el Abuso en Parejas Intragénero y Denunciarlo}

Las dificultades experimentadas por las personas entrevistadas para identificar o reconocer la violencia sufrida en una relación de pareja intragénero, tanto a nivel personal como para obtener el reconocimiento de su entorno más cercano, han sido un tema repetido en cuatro de los relatos recogidos.

Distinción entre abuso y conflicto. Una idea común entre las personas entrevistadas que identifican estas dificultades es la incapacidad inicial de identificar que están sufriendo violencia por parte de su pareja, que frecuentemente se interpreta como una discusión o conflicto entre las dos personas, como señala este participante:

Yo creo que estaba tan ciego que en ese momento no deduje un maltrato... como un maltrato muy grave. Lo veía como una discusión de parejas. ¿Cuánta importancia tiene ese golpe? Yo me di cuenta después de la importancia que tiene ese golpe. En su momento no le di esa importancia. Preferí eliminarlo y continuar con mi vida. (H, 23, Homoflexible)

Invisibilidad de la violencia intragénero. Esta dificultad de identificar que están sufriendo abuso psicológico por parte de la pareja se atribuye a la invisibilidad de la violencia intragénero y a la falta de información sobre el fenómeno para poder reconocer este tipo de violencia cuando alguien la está viviendo. Así lo expone este participante:

No fue fácil contarlo, sobre todo porque de estas cosas no se habla mucho y la gente no tiene mucho conocimiento de la violencia en parejas del mismo sexo. (H, 24, Homosexual)

De este modo, a la dificultad de identificar el abuso se le añade la dificultad de explicar dicha situación a terceras personas, dado que estas pueden no identificar la situación como abusiva, como explica esta participante:

Mis padres tampoco lo consideraron como un abuso ¿sabes? Sí que omití la parte del cabello, porque no quería más percales [conflictos], pero, en lo que se refiere al abuso psicológico, se los expliqué y ellos lo consideraron como una pelea entre amigas. (M, 25, Bisexual)

Visión heteronormativa de los roles en la pareja. La confusión entre una relación de abuso psicológico y una pelea entre amigas, por una parte, estaría relacionada con la invisibilidad del fenómeno, y, por otra, con la visión heteronormativa de los roles en la pareja. Dichos roles establecen el mito de que las mujeres no pueden ejercer violencia y los hombres no pueden ser víctimas de la misma. Este hecho podría promover que, donde podría estar ocurriendo un abuso, este se considerara una pelea entre amigas. Asimismo, la heteronormatividad estructural podría facilitar el sostenimiento de este mito, pues una relación entre mujeres sería vista en este caso como una relación entre amigas y no como una relación sexo-afectiva. En relación a este mito, otro participante señala la dificultad para asumir que otro hombre había perpetrado abuso contra él: 
Aceptar que un hombre me ha abusado... he necesitado bastante coraje. Tenía miedo al qué dirán, pero al mismo tiempo tenía deseos de hablar. Pude llegar a decir a mi grupo de amigas que esa persona con quien estaba me trataba mal o me insultaba, pero fue muy difícil. (H, 24, Homosexual)

Así, el mito que establece que las mujeres no son perpetradoras y los hombres no son víctimas podría actuar a dos niveles: individualmente, puesto que dificulta la identificación y la aceptación de la propia experiencia de abuso, y socialmente, dado que la norma heterosexual dificultaría tanto la visibilidad de las relaciones sexo-afectivas intragénero como el cuestionamiento de los roles de género, lo que puede influir en que las personas víctimas de esta violencia teman la reacción de su entorno más cercano.

Concepción del perfil de la persona maltratadora. Finalmente, otra dificultad para identificar el abuso psicológico en relaciones intragénero experimentado podría ser la creencia en la existencia de un perfil de persona maltratadora que sería agresiva o violenta. Esta participante hace el siguiente apunte al respecto:

Esta última chica era muy tranquila. Entonces, claro me cuesta ver la violencia que hubo, porque fue distinta. En un primer momento no lo piensas, porque era muy pacífica, muy sensata, con cabeza, paciente... no cuadra con perfil agresivo, pero tenía un punto [tenía una cierta agresividad]. (M, 24, Bisexual)

\section{Discusión}

\section{Estrategias de Abuso Psicológico}

El análisis de las entrevistas ha permitido identificar algunas de las estrategias de abuso psicológico más relevantes por su presencia en los relatos: el aislamiento, el control de la vida personal y algunas estrategias de abuso emocional, como el menosprecio. Estas estrategias han sido señaladas en estudios previos como relevantes en el abuso psicológico en parejas del mismo género (e.g., Bornstein et al., 2006) y de distinto género (e.g., Porrúa-García et al., 2016). Según relatan las participantes, el control de la vida personal es empleado como estrategia de abuso psicológico para someter a la pareja, como han señalado también estudios previos (Frankland \& Brown, 2014; Porrúa-García et al., 2016), siendo destacable el uso de tecnologías, como los dispositivos móviles, para ejercer dicho abuso.

Las entrevistas realizadas también destacan el aislamiento, en este caso de amistades y personas conocidas, como una de las estrategias más relevantes de abuso psicológico en relaciones intragénero (Bornstein et al., 2006). Según describen las participantes, se lleva a cabo de una forma gradual y tiene como objetivo de que su vida gire en torno a la de la persona perpetradora. El empleo del aislamiento en parejas intragénero aumenta la dependencia de las víctimas del abuso hacia las perpetradoras, limitando su capacidad de búsqueda de ayuda y de denuncia de la situación (Bornstein et al., 2006). Asimismo, el aislamiento de la pareja en el hogar fue relacionado con la LBGfobia interiorizada, que fue identificada como hostil, y tendría su origen en el estigma social hacia las personas LBG (Meyer, 2003).

Otras estrategias frecuentemente empleadas son las de abuso emocional, de acuerdo también a lo señalado en estudios previos (Mason et al., 2014; Matte \& Lafontaine, 2011), siendo destacable el menosprecio. Finalmente, es necesario destacar la amenaza de outing como estrategia específica de abuso psicológico en parejas intragénero (Balsam \& Szymanski, 2005; Woulfe \& Goodman, 2018). Al empleo de esta estrategia subyace la influencia de un entorno social que discrimina y estigmatiza a las personas no heterosexuales (Meyer, 2003) y el heterosexismo (Potoczniak et al., 2003).

\section{Explicaciones al Abuso en Parejas Intragénero}

Una de las explicaciones más relevantes aportada por las participantes es la LBGfobia interiorizada en las personas perpetradoras, a la que atribuyen el rechazo vivido por parte de las personas perpetradoras. De nuevo, la influencia del contexto social de LGTBfobia parece tener una influencia negativa sobre las relaciones de pareja intragénero (Balsam \& Szymanski, 2005; Edwards \& Sylaska, 2013).

Otra de las explicaciones que se repiten para algunas estrategias de abuso — como el aislamiento, el control de la vida personal y el abuso emocional — son los celos y la concepción de la pareja como una posesión. La sospecha de que la pareja esté teniendo otras relaciones afectivo-sexuales con otras personas es uno de los argumentos empleados por las personas perpetradoras para ejercer dichas estrategias. Cabe tener en cuenta que las personas LBG se han socializado también en unos patrones socioculturales patriarcales en los que se favorece la perpetuación de mitos relativos al amor romántico que, a su vez, fomentan conductas de 
dominación en la pareja (ver Glass, Koziol-McLain, Campbell \& Block, 2004; Rodríguez Otero \& Negroni, 2018), como podría ser el mito que identifica los celos con el amor.

Finalmente, otra de las explicaciones relatadas por las participantes es la vivencia previa de violencia, tanto por parte de la familia como de sus iguales. Las experiencias traumáticas o de violencia interpersonal aumentan las probabilidades de experimentar situaciones de violencia en el futuro (Balsam \& Szymanski, 2005), pues estas situaciones podrían facilitar el aprendizaje de pautas inadecuadas de relación que posteriormente son aplicadas en relaciones de pareja (Rey, 2002).

\section{Dificultades de Identificar el Abuso en Parejas Intragénero y la Denuncia}

El contexto de LBGfobia parece influir también en muchas de las dificultades señaladas por las participantes para identificar el abuso. Por una parte, la incapacidad de identificar el abuso, relacionada con la invisibilidad del fenómeno, la falta de información y conocimiento al respecto, y el heterosexismo que establece que las relaciones afectivo-sexuales son entre un hombre y una mujer (Potoczniak et al., 2003), dificultando, así, la consideración de relación de pareja a aquella formada por dos personas con la misma identidad de género. Por otra parte, los roles de género que establecen que las mujeres únicamente pueden ser víctimas y los hombres perpetradores también dificultarían la identificación del abuso (ver Connell \& Messerschmidt, 2005; Gillum \& DiFulvio, 2012). Estas dificultades serían una barrera más para la denuncia del abuso y la búsqueda de apoyo formal e informal (St. Pierre \& Senn, 2010).

\section{Limitaciones y Futura Investigación}

Este estudio cuenta con algunas limitaciones relativas a su muestra que es necesario destacar. En primer lugar, la muestra incluida en este estudio, a pesar de ser reducida, es diversa en cuanto a orientación afectivosexual. Sin embargo, sería conveniente en estudios futuros incluir una mayor diversidad en cuanto a la identidad de género, pues ninguna de las personas participantes tenía una identidad no binaria. En segundo lugar, la mayor parte de participantes en el estudio eran personas jóvenes residentes en un país donde el matrimonio igualitario es legal y muchas de ellas residentes en una comunidad autónoma con una ley que sanciona la LGTBIfobia y en su mayoría en núcleos urbanos. Como estudios previos han señalado, sería necesario realizar más investigación con muestras de diversas edades, en zonas con menor apoyo formal (Gillum \& DiFulvio, 2012). Asimismo, es necesario que futuros estudios sigan indagando en aquellas estrategias específicas de abuso psicológico que tienen un origen en el estigma social hacia las personas LGB, como la amenaza de outing, así como la influencia de los celos y otros mitos del amor romántico sobre la perpetración del abuso. Finalmente, es necesario mencionar que buena parte de la investigación realizada en violencia en parejas intragénero se ha realizado sobre lo sucedido durante la relación de pareja. Resulta de relevancia que futuros estudios indaguen en el abuso perpetrado una vez terminada la relación.

\section{Aportaciones Prácticas}

Estos hallazgos resultan de alta relevancia para el diseño o implementación de servicios de atención a las víctimas de violencia intragénero. Resulta imprescindible tener en cuenta el contexto de heterosexismo y de LGTBfobia en el diseño e implementación de estos servicios y de como este contexto puede dificultar la búsqueda de ayuda. Además, conocer las estrategias de abuso psicológico y las bases socioculturales en las que se apoyan para perpetrar el abuso resulta imprescindible para la prevención de dicha violencia y para la atención a las víctimas. Por último, la violencia que se ejerce sobre la pareja una vez terminada la relación influiría notablemente sobre los procesos de recuperación de las personas que han sufrido la violencia, sobre la intervención psicosocial con las personas víctimas y/o perpetradoras y sobre las medidas judiciales, en el caso de haberlas, sobre la persona perpetradora.

\section{Referencias}

Alhusen, J. L., Lucea, M. B. \& Glass, N. (2010). Perceptions of and experience with system responses to female same-sex intimate partner violence. Partner Abuse, 1, 443-462. https://doi.org/10.1891/1946-6560.1.4.443

Badenes-Ribera, L., Bonilla-Campos, A., Frias-Navarro, D., Pons-Salvador, G. \& Monterde-i-Bort, H. (2016). Intimate partner violence in self-identified lesbians: A systematic review of its prevalence and correlates. Trauma, Violence, \& Abuse, 17, 284-97. doi: $10.1177 / 1524838015584363$

Baker, N. L., Buick, J. D, Kim, S. R., Moniz, S. \& Nava, K. L. (2013). Lessons from examining same-sex intimate partner violence. Sex Roles, 69, 182-192. https://doi.org/10.1007/s11199-012-0218-3 
Balsam, K. F. \& Szymanski, D. M. (2005). Relationship quality and domestic violence in women's same-sex relationships: The role of minority stress. Psychology of Women Quarterly, 29, 258-269. https://doi.org/10.1111/j.1471-6402.2005.00220.x

Barrientos, J., Rodríguez-Carballeira, Á., Escartín, J. \& Longares, L. (2016). Violencia en parejas del mismo sexo: revisión y perspectivas actuales. Revista Argentina de Clínica Psicológica, 25, 289-298. Extraído de http://www.redalyc.org/pdf/2819/281948416008.pdf

Bartholomew, K., Regan, K. V., White, M. A. \& Oram, D. (2008). Patterns of abuse in male same-sex relationships. Violence and Victims, 23, 617-636. https://doi.org/10.1891/0886-6708.23.5.617

Bornstein, D. R., Fawcett, J., Sullivan, M., Senturia, K. D. \& Shiu-Thornton, S. (2006). Understanding the experiences of lesbian, bisexual and trans survivors of domestic violence: A qualitative study. Journal of Homosexuality, 51(1), 159-181. https://doi.org/10.1300/J082v51n01_08

Braun, V. \& Clarke, V. (2006). Using thematic analysis in psychology. Qualitative Research in Psychology, 3, 77-101. https://doi.org/10.1191/1478088706qp063oa

Carvalho, A. F., Lewis, R. J., Derlega, V. J., Winstead, B. A. \& Viggiano, C. (2011). Internalized sexual minority stressors and same-sex intimate partner violence. Journal of Family Violence, 26, 501-509. https://doi.org/10.1007/s10896-011-9384-2

Connell, R. W. \& Messerschmidt, J. W. (2005). Hegemonic masculinity: Rethinking the concept. Gender \& Society, 19, 829-859. doi: $10.1177 / 0891243205278639$

Edwards, K. M. \& Sylaska, K. M. (2013). The perpetration of intimate partner violence among LGBTQ college youth: The role of minority stress. Journal of Youth and Adolescence, 42, 1721-1731. https://doi.org/10.1007/s10964-012-9880-6

Frankland, A. \& Brown, J. (2014). Coercive control in same-sex intimate partner violence. Journal of Family Violence, 29, 15-22. https://doi.org/10.1007/s10896-013-9558-1

Gillum, T. L. \& DiFulvio, G. (2012). "There's so much at stake": Sexual minority youth discuss dating violence. Violence Against Women, 18, 725-745. https://doi.org/10.1177/1077801212455164

Glass, N., Koziol-McLain, J., Campbell, J. \& Block, C. R. (2004). Female-perpetrated femicide and attempted femicide: A case study. Violence Against Women, 10, 606-625. https://doi.org/10.1177/1077801204265016

Gómez Ojeda, F., Barrientos Delgado, J., Guzmán González, M., Cárdenas Castro, M. \& Bahamondes Correa, J. (2017). Violencia de pareja en hombres gay y mujeres lesbianas chilenas: un estudio exploratorio. Interdisciplinaria, 34, 57-72. https://doi.org/10.16888/interd.2017.34.1.4

Joffe, H. (2012). Thematic analysis. En D. Harper \& A. R. Thompson (Eds.), Qualitative research methods in mental health and psychotherapy: A guide for students and practitioners (pp. 209-223). Chichester, Reino Unido: Wiley-Blackwell.

Longares, L., Saldaña, O., Escartín, J., Barrientos, J. \& Rodríguez-Carballeira, Á. (2018a). Measuring psychological abuse in same-sex couples: Evidence of validity of the EAPA-P in a Spanish-speaking sample. Annals of Psychology, 34, 555-561. https://doi.org/10.6018/analesps.34.3.306281

Longares, L., Escartín, J., Barrientos, J. \& Rodríguez-Carballeira, Á. (2018b). Psychological abuse in Spanish same-sex couples: Prevalence and relationship between victims and perpetrators. Innovation: The European Journal of Social Science Research, 31, 125-141. https://doi.org/10.1080/13511610.2017.1326304

Mason, T. B., Lewis, R. J., Milletich, R. J., Kelley, M. L., Minifie, J. B. \& Derlega, V. J. (2014). Psychological aggression in lesbian, gay, and bisexual individuals' intimate relationships: A review of prevalence, correlates, and measurement issues. Aggression and Violent Behavior, 19, 219-234. https://doi.org/10.1016/j.avb.2014.04.001

Matte, M. \& Lafontaine, M. -F. (2011). Validation of a measure of psychological aggression in same-sex couples: Descriptive data on perpetration and victimization and their association with physical violence. Journal of GLBT Family Studies, 7, $226-244$. https://doi.org/10.1080/1550428X.2011.564944

McClennen, J. C. (2005). Domestic violence between same-gender partners: Recent findings and future research. Journal of Interpersonal Violence, 20, 149-154. https://doi.org/10.1177/0886260504268762

Meyer, I. H. (2003). Prejudice, social stress, and mental health in lesbian, gay, and bisexual populations: Conceptual issues and research evidence. Psychological Bulletin, 129, 674-697. https://doi.org/10.1037/0033-2909.129.5.674

Ortega López, A. (2014). Agresión en parejas homosexuales en España y Argentina: prevalencias y heterosexismo (Tesis de Doctorado, Universidad Complutense de Madrid, España). Extraído del Catálogo Cisne UCM-AECID: https://eprints.ucm.es/28389/1/T35737.pdf

Porrúa-García, C., Rodríguez-Carballeira, Á., Escartín, J., Gómez-Benito, J., Almendros, C. \& Martín-Peña, J. (2016). Development and validation of the Scale of Psychological Abuse in Intimate Partner Violence (EAPA-P). Psicothema, 28, 214-221. https://doi.org/10.7334/psicothema2015.197

Potoczniak, M. J., Mourot, J. E., Crosbie-Burnett, M. \& Potoczniak, D. J. (2003). Legal and psychological perspectives on same-sex domestic violence: A multisystemic approach. Journal of Family Psychology, 17, 252-259. https://doi.org/10.1037/0893-3200.17.2.252

Rey, C. (2002). Rasgos sociodemográficos e historia de maltrato en la familia de origen, de un grupo de hombres que han ejercido violencia hacia su pareja y de un grupo de mujeres víctimas de éste tipo de violencia. Revista Colombiana de Psicología, 11, 81-90. Extraído de https://revistas.unal.edu.co/index.php/psicologia/article/view/1199

Rodríguez-Carballeira, Á., Porrúa-García, C., Escartín, J., Martín-Peña, J. \& Almendros, C. (2014). Taxonomy and hierarchy of psychological abuse strategies in intimate partner relationships. Annals of Psychology, 30, 916-926. https://doi.org/10.6018/analesps.30.3.154001

Rodríguez Otero, L. M. \& Negroni, L. K. (2018). Imaginarios del amor en gays, lesbianas y bisexuales de México y España. Una investigación cualitativa. Margen, 88, 1-19. Extraído de https://www.margen.org/suscri/margen88/otero_88.pdf

St. Pierre, M. \& Senn, C. Y. (2010). External barriers to help-seeking encountered by Canadian gay and lesbian victims of intimate partner abuse: An application of the barriers model. Violence and Victims, 25, 536-552. https://doi.org/10.1891/0886-6708.25.4.536

Vaismoradi, M., Turunen, H. \& Bondas, T. (2013). Content analysis and thematic analysis: Implications for conducting a qualitative descriptive study. Nursing \& Health Sciences, 15, 398-405. https://doi.org/10.1111/nhs.12048

Walters, M. L., Chen, J. \& Breiding, M. J. (2013). The National Intimate Partner and Sexual Violence Survey (NISVS): 2010 findings on victimization by sexual orientation. Atlanta, GA: National Center for Injury Prevention and Control, Centers for Disease Control and Prevention. Extraído de http://www.cdc.gov/violenceprevention/pdf/nisvs_sofindings.pdf

Woulfe, J. M. \& Goodman, L. A. (2018). Identity abuse as a tactic of violence in LGBTQ communities: Initial validation of the Identity Abuse Measure. Journal of Interpersonal Violence. Anticipo en línea de la publicación. https://doi.org/10.1177/0886260518760018 Extraído de https://journals.sagepub.com/doi/full/10.1177/0886260518760018 
Fecha de recepción: Agosto de 2018.

Fecha de aceptación: Marzo de 2019. 